\title{
Successful Embolization Therapy through Reentry Tear in the Right Subclavian Artery for Treating Patent False Lumen in the Aortic Arch Formed after Type A Dissection Repair
}

\author{
Hirohito Ishii, MD, Kunihide Nakamura, MD, Eisaku Nakamura, MD, \\ Koji Furukawa, MD, and Kouichiro Ochiai, MD
}

\begin{abstract}
A 73-year-old woman had undergone hemiarch replacement with primary entry resection for treating acute type A dissection 6 years ago. Postoperative computed tomography $(\mathrm{CT})$ showed a patent false lumen (FL) in the aortic arch and a reentry tear in the right subclavian artery. The remaining aortic arch enlarged, which resulted in formation of a 55-mm-diameter aneurysm. We performed reentry occlusion using embolization with glue and coil. The patient's clinical course after the procedure was uneventful, and subsequent CT showed that FL was thrombosed and had decreased in size.
\end{abstract}

Keywords: acute type A aortic dissection, embolization, BCA dissection

\section{Introduction}

Surgical selection of either hemiarch replacement (HAR) or total arch replacement (TAR) for treating acute type A aortic dissection (AAAD) remains controversial. Perioperative mortality and morbidity are lower with HAR than TAR; however, HAR is associated with the potential risk of dilatation of the remaining aortic arch. ${ }^{1,2)}$ Upon postoperative enlargement of the remaining aortic arch, redoTAR can be considered as a next surgical procedure, ${ }^{1,3)}$ which has an extremely high mortality rate. ${ }^{3)}$ Therefore, other less invasive treatments are required for these clinical states. One of the potential procedures in such a situation is reentry occlusion; however, this is still uncommon in supra-aortic vessels. Here we present a case of thrombo-

Division of Cardiovascular Surgery, Department of Surgery, University of Miyazaki, Miyazaki, Japan

Received: January 31, 2017; Accepted: June 23, 2017

Corresponding author: Hirohito Ishii, MD. Division of Cardiovascular Surgery, Department of Surgery, University of Miyazaki, 5200 Kihara, Kiyotake, Miyazaki, Miyazaki 889-1692, Japan Tel: +81-985-85-2291, Fax: +81-985-85-5563

E-mail: hirohito_ishii@med.miyazaki-u.ac.jp sis and shrinkage of a false lumen (FL), which developed following AAAD repair, after embolization through a reentry tear in the right subclavian artery (RSCA).

\section{Case Report}

A 73-year-old woman developed AAAD 6 years ago. At onset, computed tomography (CT) showed a patent FL extending from the ascending aorta, which had enlarged to a diameter of $5.0 \mathrm{~cm}$, to left common iliac artery (LCIA). She underwent emergency HAR because of a primary entry tear located in the ascending aorta. Open distal anastomosis was performed under circulatory arrest. The intima and adventitia were sandwiched and reinforced with a felt strip, and the synthetic graft was secured with running sutures. Postoperative CT showed a patent FL extending from the aortic arch to LCIA and reentry tears in the RSCA (Figs. 1A and 1B), abdominal aorta, and LCIA. Three years later, aortic angiography showed strong flow from the reentry tear of RSCA to FL of the aortic arch (Fig. 1C); there were no new tears at the distal anastomosis site. Six years after onset, a follow-up CT showed a 26-mm-diameter aneurysm (enlargement of $6 \mathrm{~mm} / 6$ years) in the RSCA and a 55-mm-diameter aneurysm (enlargement of $15 \mathrm{~mm} / 6$ years) at the distal part of arch with saccular dilatation. Additionally, CT revealed a reentry tear in the RSCA, which was $2 \mathrm{~cm}$ distal to the right common carotid artery bifurcation, abdominal aorta, and LCIA. In arterial and venous phase CT, aortic arch thrombosis was detected; in addition, the area extending from abdominal aorta below the level of celiac artery to LCIA was found patent. Although CT revealed aortic arch thrombosis, we understood that reentry flow from the RSCA to FL was an important factor for aortic dilatation. We chose to perform reentry occlusion rather than redo-TAR because it is less invasive. The occlusion method involved transcatheter arterial embolization (TAE) as a distinct approach from the covered stent placement because of difficulties in per- 

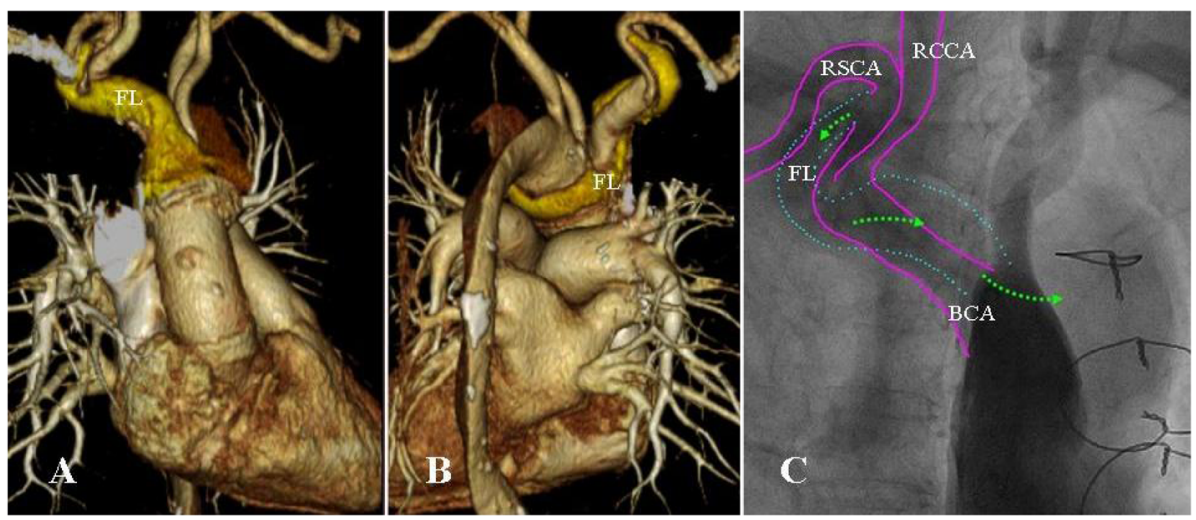

Fig. 1 Anatomic locations of reentry tears in the RSCA and subsequent FL. Threedimensional computed tomography scans taken 3 years previously show FL from the reentry tear of the RSCA to aortic arch (A is from front, B is from behind). Aortic angiography performed 3 years previously $(\mathbf{C})$ shows FL flow (arrow line) from the reentry tear of the RSCA to aortic arch. RSCA: right subclavian artery; BCA: brachiocephalic artery; RCCA: right common carotid artery; FL: false lumen

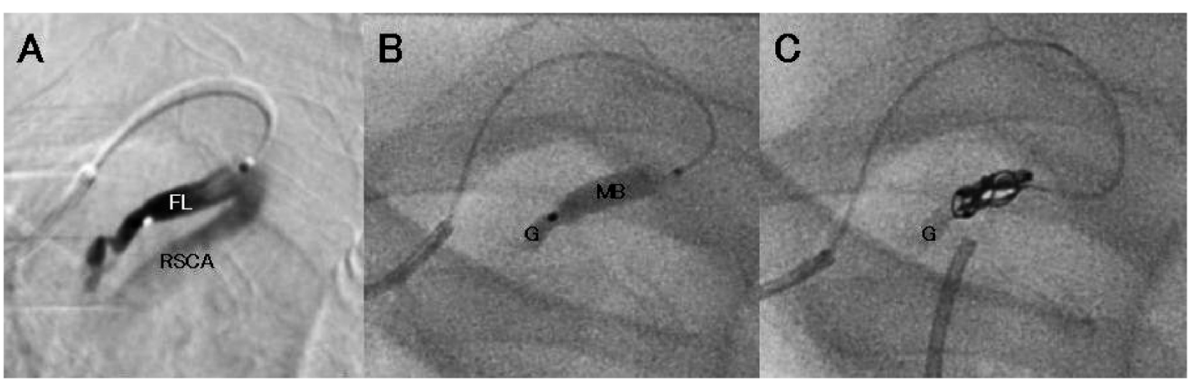

Fig. 2 Fluoroscopic images of transcatheter arterial embolization. (A) Angiography shows a reentry tear in the RSCA and FL flow. (B) Image showing "B-glue technique" involving injection of glue under microballoon occlusion. (C) Image showing an indwelling coil at the inlet port of reentry. RSCA: right subclavian artery; FL: false lumen; G: glue; MB: microballoon

forming the procedure without cervical debranching.

In TAE, the right brachial artery and right femoral artery were cannulated to obtain therapeutic and angiographic routes, respectively. Intraoperative angiography was performed, which showed reentry flow from the RSCA to FL of the brachiocephalic artery (BCA) without diffusion throughout the FL of the aortic arch (Fig. 2A). Using 0.014-inch guidewire, a 5-Fr cobra-type guiding catheter (Elway, Terumo Clinical Supply, Kakamigahara, Japan) was inserted into the RSCA and a microballoon catheter (Attendant LP, $4.5 \mathrm{~mm}$, Terumo Clinical Supply) was advanced into the FL through the tear. After microballoon occlusion of the inlet port, $2.0 \mathrm{ml}$ of $5 \%$ dextrose solution was flushed and $0.5 \mathrm{ml}$ of glue [mixture of equal parts of N-butyl-2-cyanoacrylate (NBCA) and ethiodized oil] was subsequently injected (B-glue technique) (Fig. 2B). The balloon was deflated $50 \mathrm{~s}$ after injecting the glue and removed without resistance or glue migration; afterward, a detachable microcoil $(5 \mathrm{~mm}$ diameter $\times 12 \mathrm{~cm}$ length; Cashemere, Johnson and Johnson, Tokyo, Japan) was placed proximal to the glue (Fig. 2C). The whole procedure was uneventful. CT performed immediately after TAE showed that the FL of the aortic arch was completely thrombosed, and a follow-up CT performed 2 years later showed that the FL reduced in size. Surprisingly, the FL of the abdominal aorta below the level of celiac artery was thrombosed and decreased in size (Figs. 3A-3D).

\section{Discussion}

Following surgery in patients with AAAD, aortic surgeons often face troubles of late dilatation of the distal aorta. Such dilatation after AAAD repair has been reported to occur in approximately $40 \%$ of patients. ${ }^{1,4)}$ Kimura et al. have reported that the rate of surgical reintervention (involving thoracic endovascular aneurysm repair) to the aortic arch after AAAD repair is $7.8 \%,{ }^{5)}$ and Preventza et al. have reported that the mortality rate of traditional open surgery is $11.7 \%{ }^{3)}$ In practice, distal dilatation can cause many problems, which may result in inoperable as 


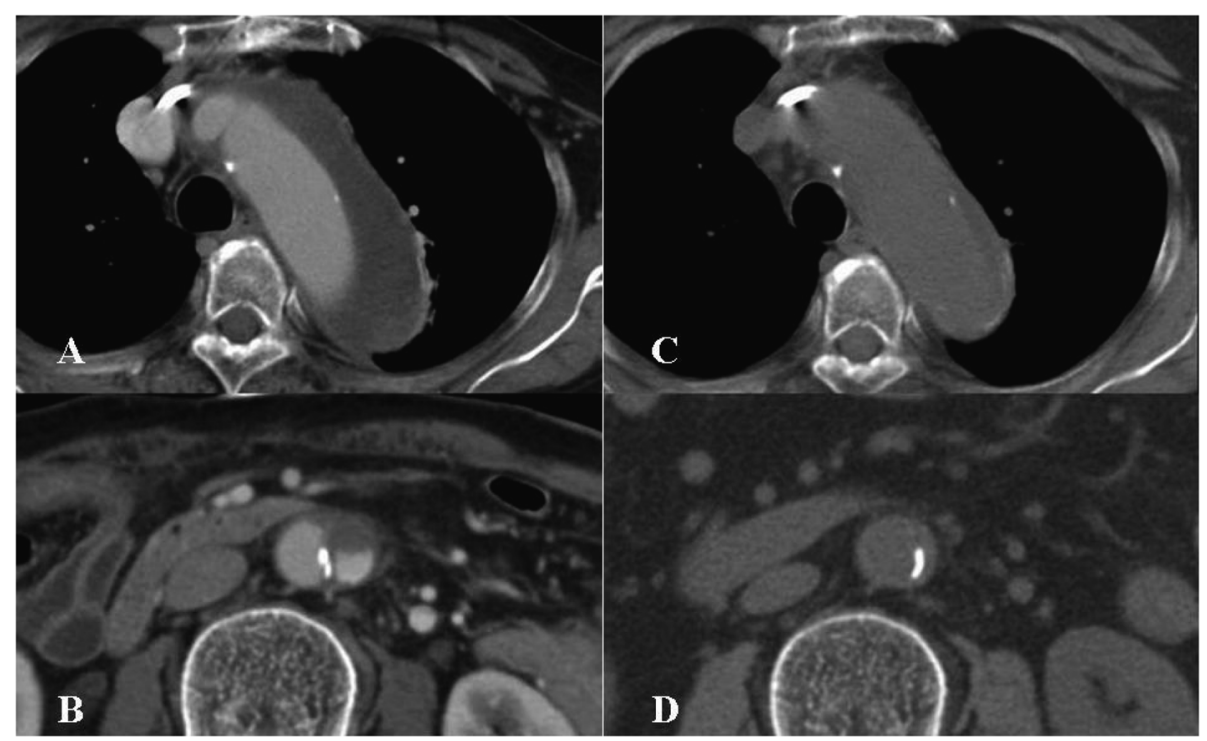

Fig. 3 Progressive computed tomography scans (axial images of the aortic arch and abdominal aorta below the level of renal artery). (A, B) Image obtained 6 years after AAAD repair but before TAE shows dilatation of the aorta; FL of the aortic arch was possibly thrombosed, and the abdominal aorta of the FL was patent. (C, D) Image obtained 2 years after TAE shows shrinkage and thrombosis of the FL. AAAD: acute type A aortic dissection; TAE: transcatheter arterial embolization; FL: false lumen

well as sudden death cases associated with aortic events. Many studies have mentioned that patent FLs and nonresection of primary entries are potential predictors of late aortic dilatation. ${ }^{1,4,5)}$ In previous AAAD cases, an intimal tear has been found in the ascending aorta in more than $60 \%$, arch in $13 \%-30 \%$, and descending aorta in $6 \%$ of cases. Additionally, multiple tears have been found in $8 \%-10 \%$ of cases, whereas $3 \%$ of cases have not shown any tear. ${ }^{5-7)}$ The primary entry tear is rarely located in the cervical branch, and entry or reentry tears are often located near the vessel ostium. Additionally, AAAD is often associated with a dissected cervical branch (BCA, 57\%; RSCA, $24 \%$; right common carotid artery, $41 \%$; left common carotid artery, $27 \%$; left subclavian artery, 24\%). ${ }^{81}$ The patency rate of FLs at the distal site after surgery is reported to be $62 \%-70 \%,{ }^{1,5,9)}$ and approximately $50 \%$ of patients with patent FLs show aortic growth. $\left.{ }^{4}\right)$ Therefore, under regulated conditions, aortic surgeons should select a surgical procedure for AAAD that rectifies problems associated with patent FLs. If patent FLs persist post-surgery, it is important to resolve them. In this context, reentry occlusion may be one of the alternative solutions; however, the use of covered stents at the site of aortic arch and cervical branch is frequently difficult without debranching of supra-aortic vessels. Additionally, Uchino et al. have described BCA dissection as a potential predictor of patent FLs of the aortic arch after HAR. ${ }^{1)}$ A previous report has mentioned that dissected BCAs remain in $48 \%$ of patients after AAAD repair. ${ }^{8}$ The BCA is larger than other branch vessels, and it receives quick and extensive blood flow from the heart. Reentry occlusion of supra-aortic vessels (especially related to the BCA) may sometimes be useful; however, it currently remains uncommon. NBCA has been used in TAE of arterial hemorrhages and aneurysms, and it has the advantage of quickly forming glue cast matching the shape of target spaces. Based on this advantage, we chose this material as an aid to coil. However, NBCA has disadvantages of proximal embolization, distal migration, reflux, etc. B-glue technique proposed by Hamaguchi et al. ${ }^{10)}$ has been used for flow control in TAE. This technique is extremely useful for preventing cerebral embolization and infarctions caused by NBCA, as observed in this case. Additionally in this case, the decisive factor for determining TAE was the angiography performed 3 years previously. It is also important to note the findings of progressive follow-up CT scans for treating aortic dissection.

\section{Conclusion}

We present a case of thrombosis and shrinkage of an FL after treatment with embolization through a reentry tear in the RSCA. Embolization of patent FLs at the BCA may be an effective therapeutic approach for treating aortic arch dilatation after HAR in patients with AAAD; additionally, it may be able to avoid redo surgeries not only in the arch but also in the thoracoabdominal aorta. Taken together, we should consider less invasive therapies, such as TAE, while selecting a surgical procedure after AAAD repair. 


\section{Acknowledgments}

The authors wish to thank Dr. Tatefumi Sakae for this excellent radiological intervention.

\section{Disclosure Statement}

The authors have no conflicts of interest to declare.

\section{Author Contributions}

Study conception: HI, KN

Data collection: $\mathrm{HI}$

Investigation: $\mathrm{HI}$

Writing: HI

Funding acquisition: HI

Critical review and revision: all authors

Final approval of the article: all authors

Accountability for all aspects of the work: all authors

\section{References}

1) Uchino G, Ohashi T, Iida $H$, et al. Predictor of patent false lumen of the aortic arch after hemiarch replacement. Gen Thorac Cardiovasc Surg 2016; 64: 722-7.

2) Rylski B, Beyersdorf F, Kari FA, et al. Acute type A aortic dissection extending beyond ascending aorta: limited or extensive distal repair. J Thorac Cardiovasc Surg 2014; 148 : 949-54; discussion, 954.

3) Preventza O, Price MD, Simpson KH, et al. Hemiarch and total arch surgery in patients with previous repair of acute type I aortic dissection. Ann Thorac Surg 2015; 100: 833-9.

4) Kimura N, Tanaka M, Kawahito K, et al. Influence of patent false lumen on long-term outcome after surgery for acute type A aortic dissection. J Thorac Cardiovasc Surg 2008; 136: 1160-6, 1166.e1-3.

5) Kimura N, Itoh S, Yuri K, et al. Reoperation of the distal aorta after initial surgery for acute type A dissection. J Thorac Cardiovasc Surg 2015; 149: S91-8.

6) Lansman SL, McCullough JN, Nguyen KH, et al. Subtypes of acute aortic dissection. Ann Thorac Surg 1999; 67: 19758; discussion, 1979-80.

7) Takami Y, Tajima K, Kato W, et al. Can we predict the site of entry tear by computed tomography in patients with acute type A aortic dissection. Clin Cardiol 2012; 35: 500-4.

8) Nagamine $H$, Ueno $Y$, Ueda $H$, et al. A new classification system for branch artery perfusion patterns in acute aortic dissection for examining the effects of central aortic repair. Eur J Cardiothorac Surg 2013; 44: 146-53.

9) Park KH, Lim C, Choi JH, et al. Midterm change of descending aortic false lumen after repair of acute type I dissection. Ann Thorac Surg 2009; 87: 103-8.

10) Hamaguchi S, Ogawa Y, Arai Y, et al. A case of pseudoaneurysm of the deep femoral artery successfully treated by NBCA embolization under occlusion. Jpn J Radiol 2013; 31 : 538-41. 